\title{
THE CONVEX HULL OF SUB-PERMUTATION MATRICES ${ }^{1}$
}

\author{
N. S. MENDELSOHN AND A. L. DULMAGE
}

1. Introduction. A combinatorial theorem $[1 ; 3]$ usually referred to as "the marriage problem" or "the problem of distinct representatives" has the following matrix formulation; the convex hull of the set of all $n$ by $n$ permutation matrices is the set of all $n$ by $n$ doubly stochastic matrices. In this note the above theorem is generalized.

The following notation and definitions will be used. $A$ will represent an $n$ by $n$ matrix with non-negative real entries $a_{i j} ; S$ will represent the sum of all entries of $A, S=\sum_{i} \sum_{j} a_{i j} ; R_{i}$ will represent the sum of the entries in the $i$ th row and $C_{j}$ will represent the sum of the entries in the $j$ th column; $M$ will represent the largest row or column sum of $A, M=\max \left(R_{i}, C_{j}\right)$. Also used will be the concept of a sub-permutation matrix of rank $r$. By this is meant a matrix $P$ with the following properties: (1) each entry of $P$ is either 1 or 0 ; (2) each row and each column of $P$ contains at most one 1 ; (3) $P$ contains exactly $r$ entries equal to 1 . In terms of this notation the theorem quoted above becomes; a matrix $A$ lies in the convex hull of the set of all permutation matrices if and only if $M=1$ and $S=n$. In [2] the authors of the present note obtain sufficient conditions in order that a matrix $A$ with non-negative entries contain nonzero entries in the places occupied by 1 in a permutation matrix of rank $r$. In this note necessary and sufficient conditions are given in order that a matrix $A$ lie in the convex hull of the sub-permutation matrices of rank $n-i(i=0,1,2, \cdots, n-1)$.

2. The TheORem. Let $A$ be an $n$ by $n$ matrix whose entries are nonnegative real numbers. $A$ necessary and sufficient condition that $A$ lie in the convex hull of all sub-permutation matrices of rank $n-i$ is that $S=n-i$ and $(n-i) / n \leqq M \leqq 1$.

Proof. The necessity is obtained as follows. Let $A=\sum_{j} \alpha_{j} P_{j}$ where $\alpha_{j} \geqq 0, \sum_{j} \alpha_{j}=1$ and $P_{j}$ is a sub-permutation matrix of rank $n-i$. Then each matrix $\alpha_{j} P_{j}$ has the sum of all its entries equal to $(n-i) \alpha_{j}$ and each row or column sum has the value $\alpha_{j}$ or 0 . Hence $S=(n-i) \sum_{j} \alpha_{j}=(n-i)$ and $M \leqq \sum_{j} \alpha_{j}=1$. Also since $n-i=S$ $=\sum_{j} R_{j} \leqq n M,(n-i) / n \leqq M$. Hence $S=n-i$ and $(n-i) / n \leqq M \leqq 1$.

To obtain the sufficiency we note that if $S=n-i$ and $(n-i) / n$

Received by the editors July 25, 1957.

1 The research reported upon here was supported in part by the National Research Council of Canada and the Mathematical Institute of the Canadian Congress of Mathematics. 
$\leqq M \leqq 1$ then $\sum R_{j}=\sum C_{j}=n-i$. Also the numbers $1-R_{1}$, $1-R_{2}, \cdots, 1-R_{n}$ are non-negative and at least one of these is positive if $i>0$. For if all of $1-R_{1}, 1-R_{2}, \cdots, 1-R_{n}$ were 0 then $R_{j}=1=M$ for all $j$ so that $S=n$ a contradiction. The matrix $A$ is now augmented to a matrix $A^{\star}$ by the addition of $i$ rows and $i$ columns as follows: $a_{r s}^{\star}=a_{r s}$ if $r$ and $s$ are both less than or equal to $n$; $a_{r s}^{\star}=0$ if $r$ and $s$ are both greater than $n ; a_{r, n+t}^{\star}=\left(1-R_{r}\right) / i$ for $r=1,2, \cdots, n ; t=1,2, \cdots, i ; a_{n+u, 0}^{\star}=\left(1-C_{v}\right) / i$ for $u=1,2, \cdots, i$; $v=1,2, \cdots, n$. The matrix $A^{\star}$ is a doubly stochastic $n+i$ by $n+i$ matrix with zeros in the lower right hand $i$ by $i$ block. By the theorem quoted in the introduction $A^{\star}=\sum \alpha_{r} P_{r}^{\star}$ where $\alpha_{r} \geqq 0, \sum \alpha_{r}=1$ and $P_{r}^{\star}$ is an $n+i$ by $n+i$ permutation matrix. Furthermore, each $P_{r}^{\star}$ has an $i$ by $i$ block of zeros in its lower right corner. Hence $P_{r}^{\star}$ has $2 i$ entries equal to 1 in its last $i$ rows and $i$ columns. If $P_{r}$ is the $n$ by $n$ matrix in the upper left hand corner of $P_{r}^{\star}, P_{r}$ contains $(n+i)-2 i$ $=n-i$ ones. Hence $P_{r}$, is a sub-permutation matrix of rank $n-i$. Also $A=\sum \alpha_{r} P_{r}$.

\section{BIBLIOGRAPHY}

1. L. Dulmage and I. Halperin, On a theorem of Fröbenius-Konig and J. von Neumann's game of hide and seek, Transactions of the Royal Society of Canada. Section III. vol. 49 (1955) pp. 23-29.

2. L. Dulmage and N. S. Mendelsohn, Some generalizations of the problem of distinct representatives, Canadian J. Math. (to appear).

3. H. B. Mann and H. J. Ryser, Systems of distinct representatives, Amer. Math. Monthly vol. 60 (1953) pp. 397-401.

UNiversity OF MANitoBa AND

Mathematics Institute, QueEn's University 